\title{
Band-gap shift of the heavily doped single- and double-donor systems $\mathrm{Si}$ :Bi and Si:P,Bi
}

\author{
C. Moysés Araújo, J. Souza de Almeida, I. Pepe, and A. Ferreira da Silva* \\ Instituto de Física, Universidade Federal da Bahia, Campus Universitário de Ondina, 40210310 Salvador, Bahia, Brazil \\ Bo E. Sernelius \\ Department of Physics and Measurement Technology, Linköping University, SE-58183 Linköping, Sweden \\ J. P. de Souza and H. Boudinov \\ Instituto de Física, Universidade Federal do Rio Grande do Sul, 91501-970 Porto Alegre, Rio Grande do Sul, Brazil
}

(Received 24 May 2000)

\begin{abstract}
The band-gap shift of the heavily single and double-donor doped systems $\mathrm{Si} B \mathrm{Bi}$ and $\mathrm{Si}: \mathrm{P}, \mathrm{Bi}$, prepared by ion implantation, was investigated theoretically and experimentally at room temperature. The calculations were carried out within a framework of the random-phase approximation and the temperature and different manybody effects were taken into account. The experimental data were obtained with photoconductivity measurements. Theoretical and experimental results fall closely together in a wide range of donor concentration.
\end{abstract}

\section{INTRODUCTION}

The investigation of the electronic properties of doubledonor semiconductor systems has attempted to describe, both qualitatively and quantitatively, the influence of such donordonor pairs mainly in the metal nonmetal (MNM) transition and in conductivity measurements. Despite the importance of the double-donors in the physics of the disordered materials, neither experimental nor theoretical efforts have been much devoted to investigating the band-gap shift (BGS), caused by heavy doping of double-donor doped systems. These systems have a merit of increasing disorder because of the different impurity ionization energies of the two dopant elements. ${ }^{1-3}$ Newman and Holcomb ${ }^{1}$ reported measurements of conductivity to determine the critical concentration for the MNM transition in Si:P,As. In the wake of recent investigation on transport properties of $\mathrm{Si}: \mathrm{P}, \mathrm{Bi},{ }^{4}$ we investigate in this work the BGS of this material as a function of impurity concentration at room temperature.

Silicon doped with bismuth is of particular interest because of its much larger ionization energy compared to other group $\mathrm{V}$ elements (P, As and $\mathrm{Sb}){ }^{4,5}$ For high enough doping concentration the donor electrons are collected at the bottom of the conduction-band valleys. There are two band gaps of interest. The energy distance between the conduction and valence-band extrema, $E_{G, 2}$, and the distance between the Fermi level and the valence-band top, $E_{G, 1}$. The former energy is called reduced band-gap energy, which can be determined from emission measurements like photoluminescence, whereas the later energy is called the optical band-gap energy. The first is equal to the band-gap value in absence of doping, $E_{G, 0}$, plus the self-energy shifts of the states at the band extrema. The second is equal to the band-gap value in absence of doping, plus the Fermi energy, plus the selfenergy shift of the state at the valence-band extremum, plus the self-energy shift of the state at the Fermi level. For the determination of the later, photoconductivity (PC) measurements were carried out here. A very illustrative way showing this scheme above is described in the work of Wagner on heavily doped silicon in Refs. 6 and 7.

The measured samples were prepared by ion implantation in van der Pauw ${ }^{8}$ structures delineated in Si chips. ${ }^{4-11}$ The optical band-gap energies obtained experimentally are compared with the ones calculated from a method based on the random-phase approximation (RPA). ${ }^{12-15}$ In this method the BGS is caused by the electron-electron and electron-impurity interactions.

\section{EXPERIMENTAL DETAILS}

Silicon wafers of $p$-type and (100) orientation with room temperature resistivities in the range of 11-25 $\Omega \mathrm{cm}$ were used for fabrication of van der Pauw devices. Multiple implantation steps of $\mathrm{Bi}$ and $\mathrm{P}$ were accumulated in the devices in order to create similar plateaulike concentration depthprofiles for $\mathrm{Bi}$ and $\mathrm{P}$ atoms, extending from the surface to a depth of $0.25 \mu \mathrm{m}$ with an estimated deviation of $\approx 5$ percent. The doses and energies of the implantation steps were determined from the transport of ions in the matter (TRIM) simulation. ${ }^{9}$ The implantation steps were performed sequentially from the highest to the lowest energy, with the $\mathrm{Bi}^{+}$ implants made prior to those of $\mathrm{P}^{+}$. The implantation process as well as the obtained values of the electron concentrations are described in detail in Ref. 4.

The experimental apparatus for PC measurements is shown schematically in Fig. 1. A halogen lamp was used as the light source for the measurements. The light beam passes through a plane diffraction gratings (varying between 1245 and $778 \mathrm{~nm}$ ), a set of lens, collimator, focal system and a second-order filter, producing a monochromatic light focused onto a sample holder assembly which is inside the dark box.

The PC signal produced is processed by using a set of low-noise amplifiers, low pass filter, and digital voltmeter. The resultant PC spectra are recorded in an acquisition system, which simultaneously displays the wavelength dependent PC intensity as shown in Figs. 2 and 3. 


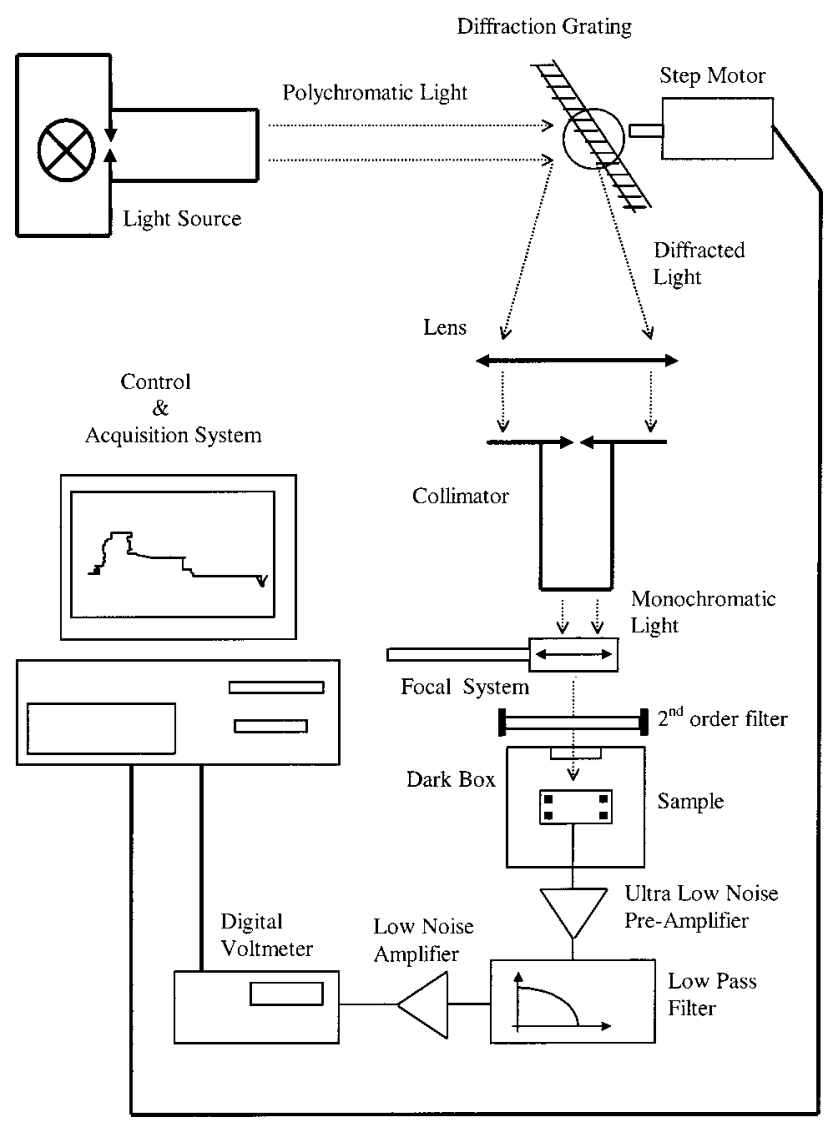

FIG. 1. The schematic experimental setup of the photoconductivity spectroscopy.

\section{RESULTS}

Figures 2 and 3 show the PC spectra of the Si:Bi and Si:P, $\mathrm{Bi}$ systems, respectively, for impurity concentration 4.0 $\times 10^{18} \mathrm{~cm}^{-3}$ and $8.3 \times 10^{19} \mathrm{~cm}^{-3}$. The insets show the determination of the threshold energy assuming the indirect transitions ${ }^{16,17}$

$$
\alpha=A\left(h v-E_{G, 1}-E_{p}\right)^{2}
$$

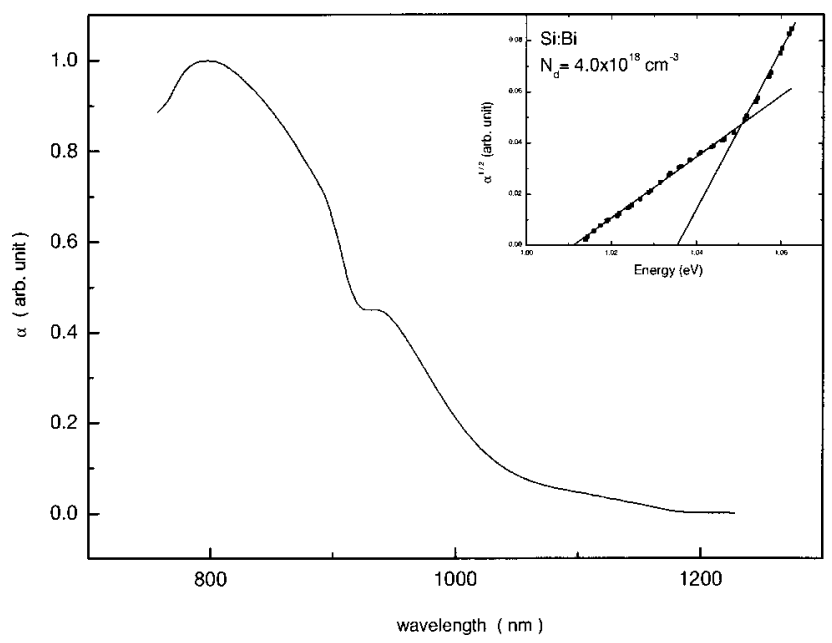

FIG. 2. Photoconductivity spectrum of $\mathrm{Si}$ :Bi with $N_{d}=4.0$ $\times 10^{18} \mathrm{~cm}^{-3}$. The insets shows the determination of the threshold energy for indirect transition. Details in the text.

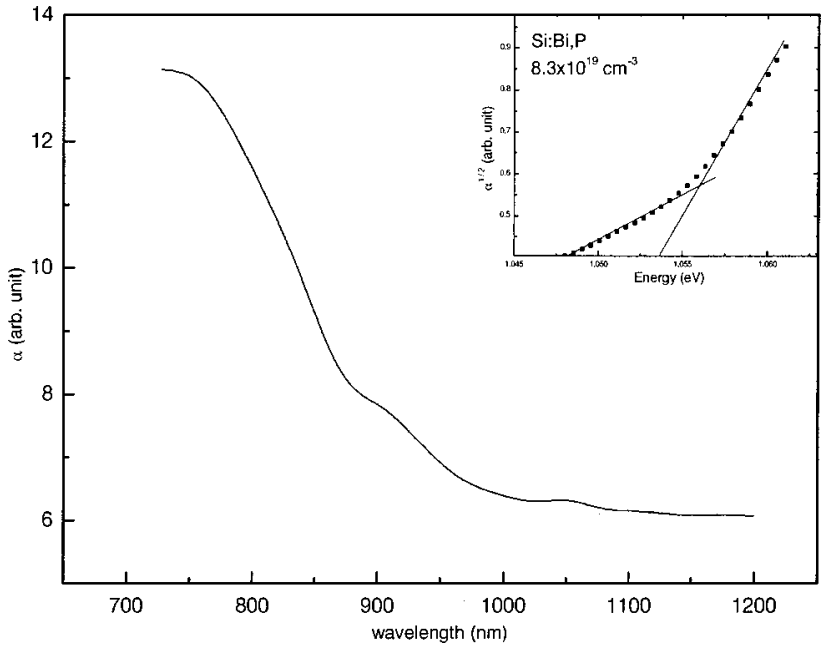

FIG. 3. Same as Fig. 2 but for Si:P, Bi with $N_{d}=8.3$ $\times 10^{19} \mathrm{~cm}^{-3}$.

where $\alpha$ is the PC intensity, $A$ a coefficient, $h v$ is the photon energy and $E_{p}$ is the phonon energy.

The value of $E_{G, 1}$ has been obtained by the extrapolation of the best-fit line between $\alpha^{1 / 2}$ and $h v$ up to the point where it crosses to the ordinary axis, as shown in the insets of Figs. 2 and 3 , but corrected by $E_{p} \cdot{ }^{17}$ The experimental values for the optical band-gap energy, as deduced from our PC measurements, are presented in Table I. In this table the values of $E_{G, 1}(\mathrm{Si}: \mathrm{P})$ are extracted from Ref. 7 and $\Delta E_{G, 1}=E_{G, 0}$ $-E_{G, 1}$, where $E_{G, 0}$ is the band-gap energy for undoped Si, here quoted as $1.115 \mathrm{eV}$, at room temperature. The overall uncertainty related to our measured values is $10 \mathrm{meV}$. The values of $\Delta E_{G, 1}$ are plotted as a function of concentration in Fig. 4 for different systems. We observe a decreasing in $\Delta E_{G, 1}$, which means an increasing in $E_{G, 1}$ in agreement with photoluminescence and photoluminescence excitation results obtained for Si:P by Wagner. ${ }^{7}$ Such results resemble the band-gap widening observed in oxides like $\operatorname{In}_{2} \mathrm{O}_{3}: \mathrm{Sn}$ and ZnO:Al. ${ }^{18,19}$

\section{THEORY AND DISCUSSION}

The different self-energy shifts presented in the determination of the two band-gap energies were calculated with many-body theory within the zero temperature formalism ${ }^{20}$ along the lines of Ref. 12. The signs of these self-energy shifts are such that all tend to reduce the band-gap values.

$\mathrm{Si}$ is a many-valley semiconductor with $v(=6)$ conduction-band minima. The band extrema are not isotropic. The Fermi volumes are ellipsoidal. We neglect this anisotropy and characterize the band dispersion with the density-of-states effective mass. Thus we represent the $v$ Fermi volumes with spheres of radii $k_{0}=\left(3 \pi^{2} n / v\right)^{1 / 3}$.

The single-particle energy for a state $(\mathbf{p}, \sigma)$ we define ${ }^{21}$ as the variational derivative of the total energy with respect to the occupation number for state $(\mathbf{p}, \sigma)$, i.e.,

$$
\varepsilon_{p}^{\sigma}=\frac{\delta(N . E)}{\delta n_{p}^{\sigma}}=\varepsilon_{p}^{0}+\hbar \Sigma_{p, \sigma},
$$

where the first term on the right hand side is the unperturbed single-particle energy (the kinetic energy) and the second, 
TABLE I. Experimental results for optical band-gap energy of differents systems. The values for Si:P are for PL and PLE measurements done by Wagner, Refs. 6 and 7, $\Delta E_{G, 1}=E_{G, 0}-E_{G, 1}$ (optical band gap), $E_{G, 0}=1.115 \mathrm{eV}$.

\begin{tabular}{|c|c|c|c|c|c|c|}
\hline $\begin{array}{l}N \\
10^{18} \mathrm{~cm}^{-3}\end{array}$ & $\begin{array}{c}\Delta E_{G, 1}(\mathrm{Si}: \mathrm{Bi}) \\
\quad(\mathrm{meV})\end{array}$ & $\begin{array}{l}\Delta E_{G, 1}(\mathrm{Si}: \mathrm{P}) \\
\quad(\mathrm{meV})\end{array}$ & $\begin{array}{l}\Delta E_{G, 1}(\mathrm{Si}: \mathrm{P}, \mathrm{Bi}) \\
\quad(\mathrm{meV})\end{array}$ & $\begin{array}{c}E_{G, 1}(\mathrm{Si}: \mathrm{Bi}) \\
(\mathrm{eV})\end{array}$ & $\begin{array}{c}E_{G, 1}(\mathrm{Si}: \mathrm{P}) \\
(\mathrm{eV})\end{array}$ & $\begin{array}{c}E_{G, 1}(\mathrm{Si}: \mathrm{P}, \mathrm{Bi}) \\
(\mathrm{eV})\end{array}$ \\
\hline 1.8 & 88 & & & 1.022 & & \\
\hline 4 & 85 & 90 & & 1.025 & 1.020 & \\
\hline 5.9 & & & 89 & & & 1.021 \\
\hline 7.6 & & & 80 & & & 1.030 \\
\hline 8.5 & & 82 & & & 1.028 & \\
\hline 15 & & 77 & & & 1.033 & \\
\hline 22 & 73 & & & 1.037 & & \\
\hline 35 & & & 70 & & & 1.040 \\
\hline 48 & 62 & & & 1.048 & & \\
\hline 50 & & 60 & & & 1.050 & \\
\hline 80 & & 54 & & & 1.056 & \\
\hline 83 & & & 59 & & & 1.051 \\
\hline 150 & & 51 & & & 1.059 & \\
\hline
\end{tabular}

the self-energy from the interactions. With the variational derivative we mean for $p<k_{0}$ minus the change in total energy when the occupation number for state $(\mathbf{p}, \sigma)$ is subtracted from the sum over occupied states; for $p>k_{0}$ we mean the change in total energy when the occupation number for state $(\mathbf{p}, \sigma)$ is added to the sum over occupied states. In other words we address the whole change in total energy to the energy of the single-particle state. This is the so-called Rayleigh-Schrödinger perturbation theory.

The two band gaps are obtained as:

$$
\begin{gathered}
E_{G, 1}=E_{G, 0}+\varepsilon_{k_{0}}^{0, e}+\hbar \Sigma_{k_{0}, \sigma}^{e}+\hbar \Sigma_{0, \sigma}^{h} ; \\
E_{G, 2}=E_{G, 0}+\hbar \Sigma_{0, \sigma}^{e}+\hbar \Sigma_{0, \sigma}^{h},
\end{gathered}
$$

where the indices $e$ and $h$ stand for electrons and holes, respectively.

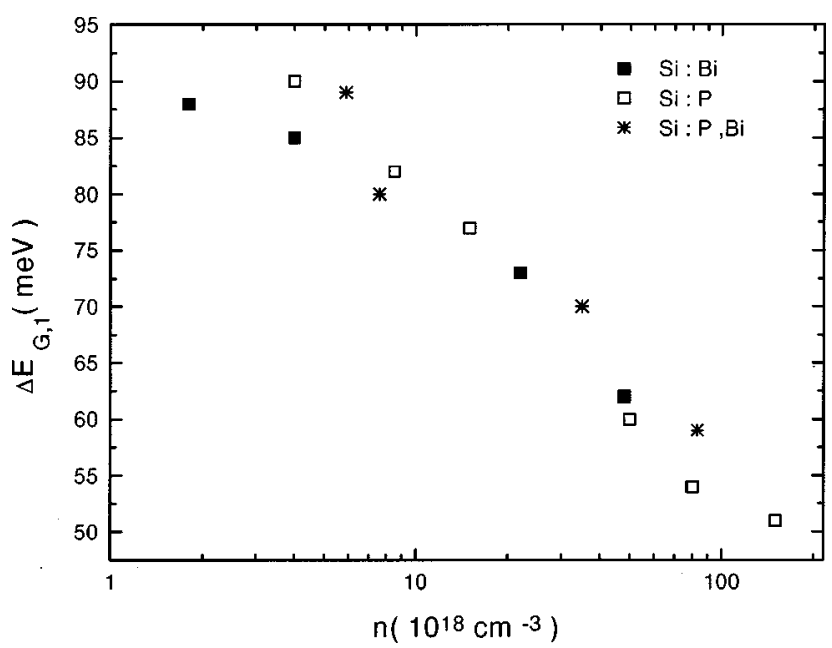

FIG. 4. Variation of $\Delta E_{G, 1}=E_{G, 0}-E_{G, 1}$ as a function of concentration, where $E_{G, 0}$ is the band energy of the undoped $\mathrm{Si}$, i.e., $E_{G, 0}=1.115 \mathrm{eV}$, and $E_{G, 1}$ is the optical band-gap energy.
We assume random distribution of donors and approximate the ionized-donor-potentials with pure Coulomb potentials. With these approximations the interaction energy consists of exchange-correlation and electron-impurityinteraction parts. The correlation energy is:

$$
\begin{aligned}
E_{c}= & +i \int_{0}^{1} \frac{d \lambda}{\lambda} \frac{1}{2 N} \sum_{q}\left\{\int_{-\infty}^{+\infty} \frac{d \omega}{2 \pi} \hbar\right. \\
& \left.\times\left[\left(\frac{1}{\varepsilon^{\lambda}(q, \omega)}-1\right)-\left(\frac{1}{\varepsilon^{H \mathrm{~F}, \lambda}(q, \omega)}-1\right)\right]\right\} ;
\end{aligned}
$$

the exchange energy is:

$$
\begin{aligned}
E_{x}= & +i \int_{0}^{1} \frac{d \lambda}{\lambda} \frac{1}{2 N} \sum_{q}\left\{\int_{-\infty}^{+\infty} \frac{d \omega}{2 \pi} \hbar\right. \\
& \left.\times\left[\left(\frac{1}{\varepsilon^{H \mathrm{~F}, \lambda}(q, \omega)}-1\right)-\left(\frac{1}{\varepsilon_{0}^{\lambda}(q, \omega)}-1\right)\right]\right\} ;
\end{aligned}
$$

the total electron-electron interaction part:

$$
\begin{aligned}
E_{\mathrm{xc}}= & +i \int_{0}^{1} \frac{d \lambda}{\lambda} \frac{1}{2 N} \sum_{q}\left\{\int_{-\infty}^{+\infty} \frac{d \omega}{2 \pi} \hbar\right. \\
& \left.\times\left[\left(\frac{1}{\varepsilon^{\lambda}(q, \omega)}-1\right)-\left(\frac{1}{\varepsilon_{0}^{\lambda}(q, \omega)}-1\right)\right]\right\} ;
\end{aligned}
$$

the electron-impurity interaction part:

$$
E_{\text {ion }}=\frac{1}{2 N} \sum_{q} \frac{n v_{q}}{k}\left(\frac{1}{\varepsilon(q, 0)}-1\right) .
$$

All these energies are energies per electron. The coupling constant $\lambda$ in Eqs. (4)-(6) is the result of the so-called ground-state energy theorem. A superscript $\lambda$ indicates that all Coulomb interactions are multiplied by $\lambda$. The dielectric function with the superscript $\mathrm{HF}$ is the dielectric function in 
the Hartree-Fock approximation. The approximation in Eq. (6) lies in the choice of dielectric function in the first term of the integrand. Choosing the Hartree-Fock dielectric function results in the exchange energy, as can be seen from Eq. (5). The second terms in $E_{\mathrm{x}}$ and $E_{\mathrm{xc}}$ represent the subtraction of the self-interaction terms and the dielectric function in these terms is an artificial function ${ }^{22}$ introduced to make the physics more transparent and simultaneously make the integrals converge faster. This dielectric function is the result where the electrons distributed over the single-particle states in the usual way, obeying the Pauli principle, but allowed to scatter into occupied states. The interested reader is referred to Ref. 22 for more details. The last energy contribution is the result to second order in the electron-impurity interaction, where $v_{q}$ is the Coulomb potential. We perform the calculation in RPA where

$$
\begin{gathered}
\varepsilon(q, \omega)=1+\alpha_{0}(q, \omega) \\
\alpha_{0}(q, \omega)=-\frac{v}{\hbar \kappa} v_{q} \sum_{\sigma} \int \frac{d^{3} k}{(2 \pi)^{3}} \\
\times \int_{-\infty}^{\infty} \frac{d \varepsilon}{2 \pi i} G_{\sigma}^{(0)}(k, \varepsilon) G_{\sigma}^{(0)}(k+q, \varepsilon+\omega) ; \\
G_{\sigma}^{(0)}(k, \varepsilon)=\frac{n_{k, \sigma}}{\varepsilon-\frac{\hbar k^{2}}{2 m^{*}}-i \eta} \quad \frac{1-\eta_{k, \sigma}}{\hbar k^{2}}+i \eta \\
n_{k, \sigma}=\left\{\begin{array}{lll}
1 & \text { if } & k<k_{0} \\
0 & \text { otherwise. }
\end{array}\right.
\end{gathered}
$$

From these relations we see how the occupation numbers enter the energy expressions. We need to know this to be able to perform the derivative in Eq. (2). To get the selfenergy shift for the valence-band holes we add a small fraction of holes; these holes give rise to an additional polarizability term in the dielectric function; this term can be expressed in terms of Green's functions for the holes in analogy with the above expression and in that way the occupation numbers for the hole states enter the energy expressions; when the functional derivatives with respect to hole occupation numbers have been taken the hole polarizabilities are let to go to zero. For more details of the calculation see Refs. 12 and $22-24$.

At finite temperatures the band-gap values change due to the following facts: The band-gap value of the undoped semiconductor decreases which leads to a reduction of both the band-gap values; the chemical potential in case of noninteracting particles moves downwards in energy which leads to a reduction of $E_{G, 1}$; the self-energy shifts are temperature dependent and this affects both band gaps. Now, this temperature dependence of the self-energy shifts has been shown to be very weak. ${ }^{25,26}$ We rely on this fact in our calculation of the band gaps. The reason for this insensitivity to temperature is the following: The shifts in the conduction band have both exchange and correlation contributions. Since a large increase in temperature leads to small occupation numbers, thus reducing the effect of the Pauli exclusion

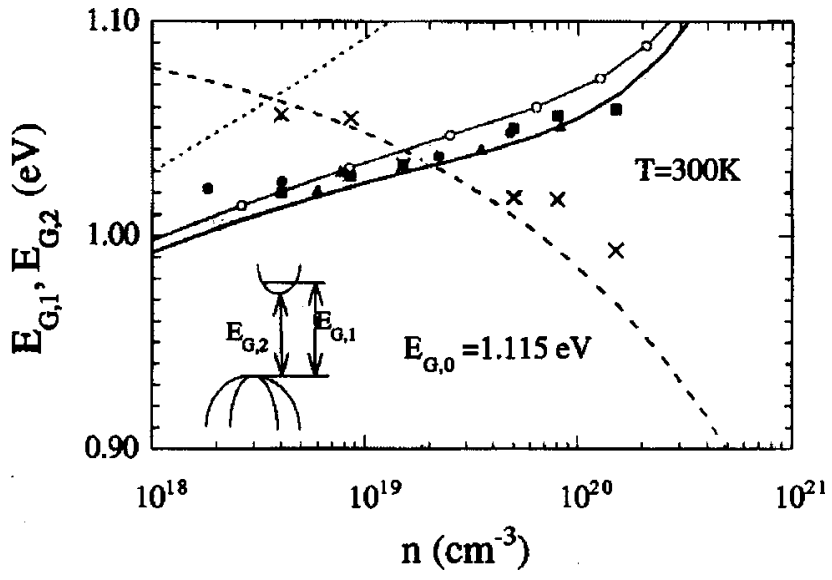

FIG. 5. The dotted, solid, and solid curve with circles are all approximations of $E_{G, 1}$. The dotted curve is the result without interactions. The solid curve is the same result corrected for the zerotemperature shifts of the states at the Fermi level and the state at the top of the valence band. The solid curve with circles is the energy distance between the chemical potential, calculated with a finite temperature formalism, and the position of the valence-band extremum with its many-body shift calculated at zero temperature. The dashed curve is $E_{G, 2}$ with zero-temperature many-body shifts. The crosses are the measured $E_{G, 2}$ values from Ref. 7. The squares are the estimated $E_{G, 1}$ values for Si:P from Ref. 6 and 7 where the many-body shifts were taken as the experimental shifts of $E_{G, 2}$. The filled circles and triangles are the experimental $E_{G, 1}$ values for $\mathrm{Si}: \mathrm{Bi}$ and $\mathrm{Si}: \mathrm{P}, \mathrm{Bi}$ as obtained from the photoconductivity measurements in the present work.

principle, the exchange contribution decreases and actually goes to zero in the limit of infinite temperature; the exchange hole is more spread out and the exchange energy is reduced. Thus one would expect a reduction of the shifts. But this spreading out of the exchange hole is compensated by a stronger screening and a restoration of hole; the correlation contribution increases and compensates to a large extent the reduction in exchange contribution. The other contributions to the self-energy shifts, the impurity contributions and also the electron-hole contribution to the shifts of the valenceband states have no exchange parts and turn out to be insensitive to temperature. The exchange-correlation hole for the states in the conduction band, the correlation hole for the states in the valence band and the screening clouds on the impurities, all contain one unit of charge at any temperature. This is probably why the temperature effects are so weak. Thus in our calculation of $E_{G, 2}$ we take the temperature effects of $E_{G, 0}$ into account only. The result is given in Fig. 5, as the dashed curve. It should be compared with the crosses which are the experimental results from luminescence experiments in Ref. 6 and 7.

For the calculation of $E_{G, 1}$, we use two different approaches. In the first approach we assume temperature independent self-energy shifts and use the zero-temperature shifts at the top of the valence band and at the Fermi wave vector together with the temperature dependent chemical potential for noninteracting particles. The result from this approach is the solid curve in Fig. 5. Neglecting many-body shifts altogether gives the dotted curve. This shift of the chemical potential is obtained implicitly from the relation 


$$
n=\int_{0}^{+\infty} d \varepsilon g(\varepsilon) \frac{1}{e^{\beta(\varepsilon-\mu)}+1} .
$$

In the second approach we calculate the chemical potential from many-body theory within the finite temperature formalism $^{20}$ along the lines of Ref. 21. In this approach we calculate the thermodynamic potential, $\Omega(T, V, \mu)$, and get the density as a function of the chemical potential from:

$$
N(T, V, \mu)=-\left(\frac{\partial \Omega}{\partial \mu}\right)_{T, V} .
$$

The thermodynamic potential can be separated into a noninteracting part, $\Omega_{0}$, and interacting parts according to

$$
\Omega(T, V, \mu)=\Omega_{0}+\Omega_{1}+\Omega_{r}+\Omega_{\text {ion }}+\ldots,
$$

where $\Omega_{1}, \Omega_{r}, \Omega_{\text {ion }}$ are the exchange part, the ring-diagram part and the contribution from the interaction with the ions, respectively. The dots represent all contributions not included here. The expressions for the thermodynamic potentials are

$$
\begin{gathered}
\Omega_{1}=\frac{1}{2} \sum_{q}\left\{\frac{\hbar}{\beta} \sum_{n}\left[\alpha_{0}\left(q, i \omega_{n}\right)\right]-v_{q} n_{0}\right\} ; \\
\Omega_{r}=\frac{1}{2} \sum_{q}\left\{\frac{\hbar}{\beta} \sum_{n}\left[\ln \left[1-\alpha_{0}\left(q, i \omega_{n}\right)\right]-\alpha_{0}\left(q, i \omega_{n}\right)\right]\right\} ; \\
\Omega_{\text {ion }}=\frac{1}{2} \sum_{q}\left\{\frac{n_{\text {ion }} v_{q}}{\kappa}\left[\frac{1}{\varepsilon(q, 0)}-1\right]\right\} ;
\end{gathered}
$$

where $n_{\text {ion }}$ is the density of donor ions, $n_{0}$ the density of electrons at the particular $\mu$ and $T$ in the absence of interactions. The polarizability is needed in the discrete set of frequencies $i \omega_{n}$, where

$$
\omega=\frac{2 n \pi}{h \beta} ; n=0, \pm 1, \pm 2, \pm 3, \ldots
$$

The polarizabilities are real valued on the imaginary frequency axis and can be obtained from the knowledge of the imaginary part of the finite temperature retarded polarizability on the real axis through the following relation:

$$
\alpha_{0}\left(q, i \omega_{n}\right)=\frac{1}{\pi} \int_{0}^{+\infty} d \omega \varepsilon_{2}^{R}(q, \omega) \frac{2 \omega}{\omega_{n}^{2}+\omega^{2}}
$$

The imaginary part of the finite temperature retarded polarizability can be obtained analytically. It is
$\varepsilon_{2}^{R}(Q, W)=-\frac{y}{2 \pi Q^{3}}\left(\frac{\pi}{4 B}\right)$

$$
\times\left[\ln \left(\frac{\cosh \left\{B\left[W+\frac{1}{2}\left(Q^{2}+\frac{W^{2}}{Q^{2}}-M\right)\right]\right\}}{\cosh \left\{B\left[W-\frac{1}{2}\left(Q^{2}+\frac{W^{2}}{Q^{2}}-M\right)\right]\right\}}\right)\right.
$$$$
-2 B W] \text {, }
$$

where

$$
\begin{gathered}
Q=\frac{q}{2 k_{0}} ; \quad W=\frac{\hbar \omega}{4 E_{0}} ; \quad E_{0}=\frac{\hbar^{2} k_{0}^{2}}{2 m^{*}} ; \\
M=\frac{\mu}{E_{0}} ; \quad B=\beta E_{0} \quad \text { and } \quad y=\frac{v m^{*} e^{2}}{\hbar^{2} \kappa k_{0}} .
\end{gathered}
$$

This approach works well as long as the density is a function of the chemical potential, i.e., as long as the chemical potential increases monotonously with density. For low temperatures this is not so and the calculation breaks down. For 300 $\mathrm{K}$ there is no problem. Also in this approach we neglect temperature effects of the shifts of the valence band. The result from this approach is given as the solid curve with circles. It is slightly above the result from the first approach. The rest of the results in Fig. 5 are experimental results for $E_{G, 1}$. The squares are the estimated values for Si:P from Ref. 6 and 7 where the many-body shifts were taken as the experimental shifts of $E_{G, 2}$. The filled circles and triangles are the experimental $E_{G, 1}$ values for $\mathrm{Si}$ Bi and $\mathrm{Si}: \mathrm{P}, \mathrm{Bi}$ as obtained from the photoconductivity measurements in the present work.

\section{SUMMARY}

We have investigated the optical band-gap energy of the heavily single and double-donor systems $\mathrm{Si}: \mathrm{Bi}$ and $\mathrm{Si}: \mathrm{P}, \mathrm{Bi}$ with photoconductivity measurements as well as with a method based on the random-phase approximation at room temperature. We have showed that, there are no strong effects of the additional vertical disorder introduced by the double doping, due to different impurity binding energies, in comparison to the two single donor materials, Si:P and Si:Bi. Experimental and theoretical results fall closely together in a wide range of impurity concentration.

\section{ACKNOWLEDGMENTS}

C.M.G.A. and J.S.A. would like to thank the Brazilian Agency, CAPES, A.F.S., J.P.S. and H.B. the Brazilian Agency CNPq and B.E.S. the Swedish Natural Science Research Council (NFR) for financial support.
*Electronic mail: ferreira@ fis.ufba.br

${ }^{1}$ P. F. Newman and D. F. Holcomb, Phys. Rev. Lett. 51, 2144 (1983).

${ }^{2}$ A. Ferreira da Silva, Phys. Rev. Lett. 59, 1263 (1987).

${ }^{3}$ A. Ferreira da Silva, Phys. Rev. B 50, 11216 (1994).

${ }^{4}$ A. Ferreira da Silva, Bo E. Sernelius, J. P. de Souza, H. Boudi- nov, H. Zheng, and M. P. Sarachik, Phys. Rev. B 60, 15824 (1999).

${ }^{5}$ E. Abramof, A. Ferreira da Silva, Bo E. Sernelius, J. P. de Souza, and H. Boudinov, Phys. Rev. B 55, 9584 (1997).

${ }^{6}$ J. Wagner, Phys. Rev. B 29, 2002 (1984); Phys. Rev. B 32, 1323 (1985). 
${ }^{7}$ J. Wagner and J. A. del Alamo, J. Appl. Phys. 63, 425 (1988).

${ }^{8}$ L. J. van der Pauw, Philips Res. Rep. 13, 1 (1958).

${ }^{9}$ J. F. Ziegler, J. P. Biersak, and U. Littmark, The Stopping and Ranges of Ions in Solids (Pergamon, New York, 1985), Vol. 1.

${ }^{10}$ J. P. de Souza and P. F. Fichtner, J. Appl. Phys. 74, 119 (1993).

${ }^{11}$ J. P. de Souza and D. K. Sadana, in Handbook on Semiconductor, edited by S. Mahajan (North-Holland, Amsterdam, 1994), Vol. 36, p. 2036.

${ }^{12}$ K.-F. Berggren and Bo E. Sernelius, Phys. Rev. B 24, 1971 (1981)

${ }^{13}$ Bo E. Sernelius, Phys. Rev. B 36, 4878 (1987).

${ }^{14}$ A. Ferreira da Silva, C. Persson, M. C. B. Marcussen, E. Veje, and A. G. de Oliveira, Phys. Rev. B 60, 2463 (1999).

${ }^{15}$ C. Persson, U. Lindefelt, and Bo E. Sernelius, Phys. Rev. B 60, 16479 (1999).

${ }^{16} \mathrm{~J}$. Tauc, in Amorphous and Liquid Semiconductors, edited by J.
Tauc (Plenum, New York, 1974), Chap. 4.

${ }^{17}$ J. I. Pankove, Optical Processes in Semiconductors (Dover, New York, 1975), Chap. 3.

${ }^{18}$ I. Hamberg, C. G. Granqvist, K.-F. Berggren, Bo E. Sernelius, and L. Engstrom, Phys. Rev. B 30, 3240 (1984).

${ }^{19}$ Bo E. Sernelius, K.-F. Berggren, Z.-C. Jin, I. Hamberg and C. G. Granqvist, Phys. Rev. B 37, 10244 (1988).

${ }^{20}$ G. D. Mahan, Many-Particle Physics, 2nd Ed. (Plenum, New York, 1990).

${ }^{21}$ T. M. Rice, Ann. Phys. (Leipzig) 31, 100 (1965).

${ }^{22}$ Bo E. Sernelius, Phys. Rev. B 34, 5610 (1986).

${ }^{23}$ Bo E. Sernelius, Phys. Rev. B 33, 8582 (1986).

${ }^{24}$ Bo E. Sernelius, Phys. Rev. B 34, 8696 (1986).

${ }^{25}$ Bo E. Sernelius, Inst. Phys. Conf. Ser. 95, 137 (1989).

${ }^{26}$ F. Thuselt and M. Rosler, Phys. Status Solidi B 130, 661 (1985). 